\title{
Some new integral inequalities on time scales containing integration on infinite intervals
}

\author{
Zhaowei Meng, Bin Zheng* and Chuanbao Wen
}

"Correspondence:

zhengbin2601@126.com

School of Science, Shandong

University of Technology,

Zhangzhou Road 12, Zibo,

Shandong 255049, China

\begin{abstract}
In this paper, some new Gronwall-Bellman type integral inequalities on time scales containing integration on infinite intervals are established. They provide new bounds for unknown functions concerned and can be used as a handy tool in the research of qualitative and quantitative properties of solutions of certain dynamic equations on time scales.
\end{abstract}

MSC: 26E70; 26D15; 26D10

Keywords: integral inequalities; time scales; dynamic equation; quantitative properties; qualitative properties

\section{Introduction}

The development of the theory of time scales was initiated by Hilger [1] as a theory capable to contain both difference and differential calculus in a consistent way. Since then many authors have expounded on various aspects of the theory of dynamic equations on time scales (for example, see [2-9], and the references therein). In these investigations, integral inequalities on time scales have been paid much attention by many authors, and a lot of integral inequalities on time scales have been established (for example, see [5-16] and the references therein), which are mainly designed to unify continuous and discrete analysis and play an important role in the research of boundedness, uniqueness, stability of solutions of dynamic equations on time scales. Among these inequalities, Gronwall-Bellman type integral inequalities are of particular importance as such inequalities provide explicit bounds for unknown functions. For related results, we refer to [10-16]. But to our knowledge, Gronwall-Bellman type integral inequalities on time scales containing integration on infinite intervals have been paid little attention so far in the literature.

In this paper, we establish some new Gronwall-Bellman type integral inequalities on time scales containing integration on infinite intervals. New explicit bounds for unknown functions concerned are obtained due to the presented inequalities. Also we present some applications for the established results.

\section{Some preliminaries}

Throughout this paper, $\mathbb{R}$ denotes the set of real numbers and $\mathbb{R}_{+}=[0, \infty)$, while $\mathbb{Z}$ denotes the set of integers. For two given sets $G, H$, we denote the set of maps from $G$ to $H$ by $(G, H)$.

๑ 2013 Meng et al.; licensee Springer. This is an Open Access article distributed under the terms of the Creative Commons Attribution License (http://creativecommons.org/licenses/by/2.0), which permits unrestricted use, distribution, and reproduction in any medium, provided the original work is properly cited. 
A time scale is an arbitrary nonempty closed subset of real numbers. In this paper, $\mathbb{T}$ denotes an arbitrary time scale. On $\mathbb{T}$ we define the forward and backward jump operators $\sigma \in(\mathbb{T}, \mathbb{T})$ and $\rho \in(\mathbb{T}, \mathbb{T})$ such that $\sigma(t)=\inf \{s \in \mathbb{T}, s>t\}, \rho(t)=\sup \{s \in \mathbb{T}, s<t\}$.

Definition 2.1 The graininess $\mu \in\left(\mathbb{T}, \mathbb{R}_{+}\right)$is defined by $\mu(t)=\sigma(t)-t$.

Definition 2.2 The cylinder transformation $\xi_{h}$ is defined by

$$
\xi_{h}(z)= \begin{cases}\frac{\log (1+h z)}{h} & \text { if } h \neq 0\left(\text { for } z \neq-\frac{1}{h}\right) \\ z & \text { if } h=0\end{cases}
$$

where Log is the principal logarithm function.

Definition 2.3 For $p \in \Re$, the exponential function is defined by

$$
e_{p}(t, s)=\exp \left(\int_{s}^{t} \xi_{\mu(\tau)}(p(\tau)) \Delta \tau\right) \quad \text { for } s, t \in \mathbb{T} \text {. }
$$

Definition 2.4 If $\sup _{t \in \mathbb{T}} t=\infty, p \in \Re$, we define

$$
e_{p}(\infty, s)=\exp \left(\int_{s}^{\infty} \xi_{\mu(\tau)}(p(\tau)) \Delta \tau\right) \quad \text { for } t \in \mathbb{T} \text {. }
$$

Remark 2.1 If $\mathbb{T}=\mathbb{R}$, then we have

$$
\left\{\begin{array}{l}
e_{p}(t, s)=\exp \left(\int_{s}^{t} p(\tau) d \tau\right) \quad \text { for } s, t \in \mathbb{R} \\
e_{p}(\infty, s)=\exp \left(\int_{s}^{\infty} p(\tau) d \tau\right) \quad \text { for } s \in \mathbb{R} .
\end{array}\right.
$$

If $\mathbb{T}=\mathbb{Z}$, then we have

$$
\left\{\begin{array}{l}
e_{p}(t, s)=\prod_{\tau=s}^{t-1}[1+p(\tau)] \quad \text { for } s, t \in \mathbb{Z} \text { and } s<t, \\
e_{p}(\infty, s)=\prod_{\tau=s}^{\infty}[1+p(\tau)] \quad \text { for } s \in \mathbb{Z} .
\end{array}\right.
$$

The following two theorems include some known properties on the exponential function.

Theorem 2.1 [17, Theorem 5.2] If $p \in \mathfrak{R}$, then the following conclusions hold:

(i) $e_{p}(t, t) \equiv 1$ and $e_{0}(t, s) \equiv 1$,

(ii) $e_{p}(\sigma(t), s)=(1+\mu(t) p(t)) e_{p}(t, s)$,

(iii) if $p \in \mathfrak{R}^{+}$, then $e_{p}(t, s)>0, \forall s, t \in \mathbb{T}$,

(iv) if $p \in \mathfrak{R}^{+}$, then $\ominus p \in \mathfrak{R}^{+}$,

(v) $e_{p}(t, s)=\frac{1}{e_{p}(s, t)}=e_{\ominus p}(s, t)$,

where $\ominus p=-\frac{p}{1+\mu p}$.

Remark 2.2 If $s=\infty$, then Theorem 2.1(iii), (v) still holds. 
Theorem 2.2 [17, Theorem 5.1] If $p \in \mathfrak{R}$, and fix $t_{0} \in \mathbb{T}$, then the exponential function $e_{p}\left(t, t_{0}\right)$ is the unique solution of the following initial value problem:

$$
\left\{\begin{array}{l}
y^{\Delta}(t)=p(t) y(t) \\
y\left(t_{0}\right)=1
\end{array}\right.
$$

For more details about the calculus of time scales, we refer to [18, 19].

The following lemma is important for proving our results.

Lemma 2.1 Suppose that $\sup _{t \in \mathbb{T}^{\kappa}} t=\infty, u, q \in C_{\mathrm{rd}}, p \in \mathfrak{R}^{+}$, and $u$ is delta differential at $t \in \mathbb{T}^{\kappa}$, then

$$
u^{\Delta}(t) \geq p(t) u(t)-q(t), \quad t \in \mathbb{T}^{\kappa}
$$

implies

$$
u(t) \leq u(\infty) e_{\ominus p}(\infty, t)+\int_{t}^{\infty} q(s) e_{p}(t, \sigma(s)) \Delta s, \quad t \in \mathbb{T}^{\kappa}
$$

Proof Since $p \in \mathfrak{R}^{+}$, then from Theorem 2.1(iv) we have $\ominus p \in \mathfrak{R}^{+}$, and furthermore, from Theorem 2.1(iii) we obtain $e_{\ominus p}(t, \alpha)>0, \forall \alpha \in \mathbb{T}^{\kappa}$.

So,

$$
\left[u(t) e_{\ominus p}(t, \alpha)\right]^{\Delta}=\left[e_{\ominus p}(t, \alpha)\right]^{\Delta} u(t)+e_{\ominus p}(\sigma(t), \alpha) u^{\Delta}(t)
$$

On the other hand, from Theorem 2.2 we have

$$
\left[e_{\ominus p}(t, \alpha)\right]^{\Delta}=(\ominus p)(t) e_{\ominus p}(t, \alpha)
$$

So, combining (2.3), (2.4) and Theorem 2.1, it follows that

$$
\begin{aligned}
{\left[u(t) e_{\ominus p}(t, \alpha)\right]^{\Delta} } & =(\ominus p)(t) e_{\ominus p}(t, \alpha) u(t)+e_{\ominus p}(\sigma(t), \alpha) u^{\Delta}(t) \\
& =e_{\ominus p}(\sigma(t), \alpha)\left[\frac{(\ominus p)(t)}{1+\mu(t)(\ominus p)(t)} u(t)+u^{\Delta}(t)\right] \\
& =e_{\ominus p}(\sigma(t), \alpha)\left[u^{\Delta}(t)-p(t) u(t)\right] .
\end{aligned}
$$

Substituting $t$ with $s$ and an integration for (2.5) with respect to $s$ from $\alpha$ to $\infty$ yield

$$
u(\infty) e_{\ominus p}(\infty, \alpha)-u(\alpha) e_{\ominus p}(\alpha, \alpha)=\int_{\alpha}^{\infty} e_{\ominus p}(\sigma(s), \alpha)\left[u^{\Delta}(s)-p(s) u(s)\right] \Delta s
$$

Since $e_{\ominus p}(\alpha, \alpha)=1$, from (2.1) and (2.6) we have

$$
u(\infty) e_{\ominus p}(\infty, \alpha)-u(\alpha) \geq-\int_{\alpha}^{\infty} e_{\ominus p}(\sigma(s), \alpha) q(s) \Delta s=-\int_{\alpha}^{\infty} e_{p}(\alpha, \sigma(s)) q(s) \Delta s
$$


which is followed by

$$
u(\alpha) \leq u(\infty) e_{\ominus p}(\infty, \alpha)+\int_{\alpha}^{\infty} e_{p}(\alpha, \sigma(s)) q(s) \Delta s
$$

Since $\alpha \in \mathbb{T}^{\kappa}$ is arbitrary, after substituting $\alpha$ with $t$, we obtain the desired inequality.

Lemma $2.2[20]$ Assume that $a \geq 0, p \geq q \geq 0$, and $p \neq 0$, then for any $K>0$

$$
a^{\frac{q}{p}} \leq \frac{q}{p} K^{\frac{q-p}{p}} a+\frac{p-q}{p} K^{\frac{q}{p}}
$$

\section{Main results}

Theorem 3.1 Suppose that $\sup _{t \in \mathbb{T}^{k}} t=\infty, u, a, f, g, h \in C_{\mathrm{rd}}\left(\mathbb{T}, \mathbb{R}_{+}\right)$, and a is decreasing. If $u(t)$ satisfies the following inequality:

$$
u(t) \leq a(t)+\int_{t}^{\infty}\left[f(s) u(s)+g(s) \int_{s}^{\infty} h(\xi) u(\xi) \Delta \xi\right] \Delta s, \quad t \in \mathbb{T}^{\kappa}
$$

then

$$
u(t) \leq a(t) e_{\ominus \tilde{f}}(\infty, t), \quad t \in \mathbb{T}^{\kappa},
$$

provided that $1+\mu(t) \tilde{f}(t)>0$, where

$$
\tilde{f}(t)=-\left[f(t)+g(t) \int_{t}^{\infty} h(\xi) \Delta \xi\right] .
$$

Proof Fix $T \in \mathbb{T}^{\kappa}$, and let $t \in[T, \infty) \cap \mathbb{T}^{\kappa}$. Since $a(t)$ is decreasing on $\mathbb{T}^{\kappa}$, from (3.1) we have

$$
u(t) \leq a(T)+\int_{t}^{\infty}\left[f(s) u(s)+g(s) \int_{s}^{\infty} h(\xi) u(\xi) \Delta \xi\right] \Delta s, \quad t \in[T, \infty) \cap \mathbb{T}^{\kappa} .
$$

Denote the right-hand side of (3.4) by $v(t)$. Then it follows that

$$
u(t) \leq v(t), \quad t \in[T, \infty) \cap \mathbb{T}^{\kappa} ;
$$

and furthermore,

$$
\begin{aligned}
v^{\Delta}(t) & =-\left[f(t) u(t)+g(t) \int_{t}^{\infty} h(\xi) u(\xi) \Delta \xi\right] \\
& \geq-\left[f(t)+g(t) \int_{t}^{\infty} h(\xi) \Delta \xi\right] v(t)=\widetilde{f}(t) v(t),
\end{aligned}
$$

where $\widetilde{f}$ is defined in (3.3).

Under the condition $1+\mu(t) \widetilde{f}(t)>0$, in fact we have $\widetilde{f} \in \mathfrak{R}^{+}$. So, a suitable application of Lemma 2.1 yields

$$
v(t) \leq v(\infty) e_{\ominus \tilde{f}}(\infty, t), \quad t \in[T, \infty) \cap \mathbb{T}^{\kappa} .
$$


Since $v(\infty)=a(T)$, then (3.7) can be rewritten as

$$
v(t) \leq a(T) e_{\ominus \tilde{f}}(\infty, t), \quad t \in[T, \infty) \cap \mathbb{T}^{\kappa} .
$$

Combining (3.8) with (3.5), we have

$$
u(t) \leq a(T) e_{\ominus \widetilde{f}}(\infty, t), \quad t \in[T, \infty) \cap \mathbb{T}^{\kappa} .
$$

Setting $t=T$ in (3.9), since $T$ is selected from $\mathbb{T}^{\kappa}$ arbitrarily, after substituting $T$ with $t$, we obtain the desired inequality (3.2).

Since $\mathbb{T}$ is an arbitrary time scale, if we take $\mathbb{T}$ for some peculiar cases, then we have the following corollaries.

Corollary 3.1 Suppose that $\mathbb{T}=\mathbb{R} . u, a, f, g, h \in C\left(\mathbb{R}, \mathbb{R}_{+}\right)$, and $a$ is decreasing on $\mathbb{R}$. If $u(t)$ satisfies the following inequality:

$$
u(t) \leq a(t)+\int_{t}^{\infty}\left[f(s) u(s)+g(s) \int_{s}^{\infty} h(\xi) u(\xi) d \xi\right] d s, \quad t \in \mathbb{R}
$$

then

$$
u(t) \leq a(t) \exp \left(-\int_{t}^{\infty} \tilde{f}(\tau) d \tau\right), \quad t \in \mathbb{R}
$$

where $\widetilde{f}(t)=-\left[f(t)+g(t) \int_{t}^{\infty} h(\xi) d \xi\right]$.

Proof When $\mathbb{T}=\mathbb{R}$, we have $\mu(t)=\sigma(t)-t \equiv 0$, and then $\ominus \widetilde{f}=-\frac{\tilde{f}}{1+\mu f}=-\tilde{f}$. Obviously, $1+\mu(t) \tilde{f}(t)=1>0$. Furthermore, from Remark 2.1 we have

$$
e_{\ominus \tilde{f}}(\infty, t)=\exp \left(\int_{t}^{\infty}(\ominus \tilde{f})(\tau) d \tau\right)=\exp \left(-\int_{t}^{\infty} \tilde{f}(\tau) d \tau\right)
$$

Combining (3.12) and (3.2), we obtain the desired result.

Corollary 3.2 Suppose that $\mathbb{T}=\mathbb{Z} . u, a, f, g, h \in\left(\mathbb{Z}, \mathbb{R}_{+}\right)$, and $a$ is decreasing on $\mathbb{Z}$. If $u(n)$ satisfies the following inequality:

$$
u(n) \leq a(n)+\sum_{s=n}^{\infty}\left[f(s) u(s)+g(s) \sum_{\xi=s}^{\infty} h(\xi) u(\xi)\right], \quad n \in \mathbb{Z},
$$

then

$$
u(n) \leq a(n) \prod_{\tau=n}^{\infty} \frac{1}{1+\widetilde{f}(\tau)}, \quad n \in \mathbb{Z}
$$

provided that $1+\tilde{f}(n)>0$, where $\tilde{f}(n)=-\left[f(n)+g(n) \sum_{\xi=n}^{\infty} h(\xi)\right]$. 
Proof When $\mathbb{T}=\mathbb{Z}$, we have $\mu(n)=\sigma(n)-n \equiv 1, \forall n \in \mathbb{Z}$, and then $\ominus \widetilde{f}=-\frac{\widetilde{f}}{1+\mu \tilde{f}}=-\frac{\tilde{f}}{1+f}$. Furthermore, from Remark 2.1 we have

$$
e_{\ominus \tilde{f}}(\infty, n)=\prod_{\tau=n}^{\infty}[1+(\ominus \tilde{f})(\tau)]=\prod_{\tau=n}^{\infty} \frac{1}{1+\widetilde{f}(\tau)}
$$

Combining (3.15) and (3.2), we obtain the desired result.

Theorem 3.2 Suppose that $\sup _{t \in \mathbb{T}^{\kappa}} t=\infty, u, a, f, g$ are defined as in Theorem 3.1. $p$ is a positive number with $p \geq 1$. If $u(t)$ satisfies the following inequality:

$$
u^{p}(t) \leq a(t)+\int_{t}^{\infty}[f(s) u(s)+g(s)] \Delta s, \quad t \in \mathbb{T}^{\kappa},
$$

then

$$
u(t) \leq\left\{a(t) e_{\ominus \widehat{f}}(\infty, t)+\int_{t}^{\infty} \widehat{g}(s) e_{\widehat{f}}(t, \sigma(s)) \Delta s\right\}^{\frac{1}{p}}, \quad t \in \mathbb{T}^{\kappa},
$$

provided $1+\mu(t) \widehat{f}(t)>0$, where

$$
\left\{\begin{array}{l}
\widehat{f}(t)=-f(t) \frac{1}{p} K^{\frac{1-p}{p}} \\
\widehat{g}(t)=f(t) \frac{p-1}{p} K^{\frac{1}{p}}+g(t), \quad \forall K>0 .
\end{array}\right.
$$

Proof Fix $T \in \mathbb{T}^{\kappa}$. Let $t \in[T, \infty) \cap \mathbb{T}^{\kappa}$, and

$$
v(t)=a(T)+\int_{t}^{\infty}[f(s) u(s)+g(s)] \Delta s, \quad t \in \mathbb{T}^{\kappa} .
$$

Then

$$
u(t) \leq v^{\frac{1}{p}}(t), \quad t \in[T, \infty) \cap \mathbb{T}^{\kappa},
$$

and furthermore,

$$
v^{\Delta}(t)=-[f(t) u(t)+g(t)] \geq-\left[f(t) v^{\frac{1}{p}}(t)+g(t)\right], \quad t \in[T, \infty) \cap \mathbb{T}^{\kappa} .
$$

On the other hand, from Lemma 2.2 we have

$$
\nu^{\frac{1}{p}}(t) \leq \frac{1}{p} K^{\frac{1-p}{p}} \nu(t)+\frac{p-1}{p} K^{\frac{1}{p}}, \quad \forall K>0 .
$$

So, combining (3.21) and (3.22), we obtain

$$
\begin{aligned}
v^{\Delta}(t) & \geq-\left[f(t)\left(\frac{1}{p} K^{\frac{1-p}{p}} v(t)+\frac{p-1}{p} K^{\frac{1}{p}}\right)+g(t)\right] \\
& =\widehat{f}(t) v(t)-\widehat{g}(t), \quad t \in[T, \infty) \cap \mathbb{T}^{\kappa},
\end{aligned}
$$

where $\widehat{f}$ and $\widehat{g}$ are defined in (3.18). 
Since $v(\infty)=a(T)$, and $1+\mu(t) \widehat{f}(t)>0$, we have $\widehat{f}(t) \in \mathfrak{R}^{+}$, and a suitable application of Lemma 2.1 yields

$$
v(t) \leq a(T) e_{\ominus \widehat{f}}(\infty, t)+\int_{t}^{\infty} \widehat{g}(s) e_{\widehat{f}}(t, \sigma(s)) \Delta s, \quad t \in[T, \infty) \cap \mathbb{T}^{\kappa} .
$$

Combining (3.24) and (3.20), we have

$$
u(t) \leq\left\{a(T) e_{\ominus \widehat{f}}(\infty, t)+\int_{t}^{\infty} \widehat{g}(s) e_{\widehat{f}}(t, \sigma(s)) \Delta s\right\}^{\frac{1}{p}}, \quad t \in[T, \infty) \cap \mathbb{T}^{\kappa}
$$

Setting $t=T$ in (3.25), and considering $T$ is selected from $\mathbb{T}^{\kappa}$ arbitrarily, after substituting $T$ with $t$, we obtain the desired inequality (3.17).

Corollary 3.3 Suppose that $\mathbb{T}=\mathbb{R} . u, a, f, g \in C\left(\mathbb{R}, \mathbb{R}_{+}\right)$, and $a$ is decreasing on $\mathbb{R}$. $p$ is defined as in Theorem 3.2. If $u(t)$ satisfies the following inequality:

$$
u^{p}(t) \leq a(t)+\int_{t}^{\infty}[f(s) u(s)+g(s)] d s, \quad t \in \mathbb{R},
$$

then

$$
u(t) \leq\left\{a(t) \exp \left(-\int_{t}^{\infty} \widehat{f}(\tau) d \tau\right)+\int_{t}^{\infty} \widehat{g}(s) \exp \left(\int_{s}^{t} \widehat{f}(\tau) d \tau\right) d s\right\}^{\frac{1}{p}}, \quad t \in \mathbb{R},
$$

where $\widehat{f}, \widehat{g}$ are the same as in Theorem 3.2 .

Corollary 3.4 Suppose that $\mathbb{T}=\mathbb{Z} . u, a, f, g \in\left(\mathbb{Z}, \mathbb{R}_{+}\right)$, and a is decreasing on $\mathbb{Z} \cdot p$ is defined as in Theorem 3.2. If $u(n)$ satisfies the following inequality:

$$
u^{p}(n) \leq a(n)+\sum_{s=n}^{\infty}[f(s) u(s)+g(s)], \quad n \in \mathbb{Z},
$$

then

$$
u(n) \leq\left\{a(n) \prod_{\tau=n}^{\infty} \frac{1}{1+\widehat{f}(\tau)}+\sum_{s=n}^{\infty} \widehat{g}(s)\left(\prod_{\tau=n}^{s} \frac{1}{1+\widehat{f}(\tau)}\right)\right\}^{\frac{1}{p}}, \quad n \in \mathbb{Z},
$$

where $\widehat{f}, \widehat{g}$ are the same as in Theorem 3.2.

The proofs for Corollaries 3.3 and 3.4 are similar to those for Corollaries 3.1 and 3.2, and we omit them here.

Theorem 3.3 Suppose that $\sup _{t \in \mathbb{T}^{k}} t=\infty, u, a, f, g$, h are defined as in Theorem 3.1, and $p \geq 1$. If $u(t)$ satisfies the following inequality:

$$
u^{p}(t) \leq a(t)+\int_{t}^{\infty}\left[f(s) u^{p}(s)+g(s) u(s)+h(s)\right] \Delta s, \quad t \in \mathbb{T}^{\kappa},
$$


then

$$
u(t) \leq\left\{\left[a(t) e_{\ominus H_{1}}(\infty, t)+\int_{t}^{\infty} H_{2}(s) e_{H_{1}}(t, \sigma(s)) \Delta s\right] e_{\ominus(-f)}(\infty, t)\right\}^{\frac{1}{p}}
$$

provided that $1-\mu(t) f(t)>0,1+\mu(t) H_{1}(t)>0$, where

$$
\left\{\begin{array}{l}
H_{1}(t)=-g(t) \frac{1}{p} K^{\frac{1-p}{p}} e_{\ominus(-f)}(\infty, t), \\
H_{2}(t)=g(t) \frac{p-1}{p} K^{\frac{1}{p}}+h(t), \quad \forall K>0 .
\end{array}\right.
$$

Proof Let

$$
v(t)=a(t)+\int_{t}^{\infty}[g(s) u(s)+h(s)] \Delta s, \quad t \in \mathbb{T}^{\kappa} .
$$

Then

$$
u^{p}(t) \leq v(t)+\int_{t}^{\infty} f(s) u^{p}(s) \Delta s, \quad t \in \mathbb{T}^{\kappa}
$$

Since $1-\mu(t) f(t)>0$, we have $-f \in \mathfrak{R}^{+}$, and furthermore $\ominus(-f) \in \mathfrak{R}^{+}$. Treating $u^{p}(t)$ as a variable, by Corollary 3.5 we obtain

$$
u^{p}(t) \leq v(t) e_{\ominus(-f)}(\infty, t), \quad t \in \mathbb{T}^{\kappa}
$$

Now fix $T \in \mathbb{T}^{\kappa}$. Let $t \in[T, \infty) \cap \mathbb{T}^{\kappa}$, and

$$
y(t)=a(T)+\int_{t}^{\infty}[g(s) u(s)+h(s)] \Delta s, \quad t \in \mathbb{T}^{\kappa} .
$$

Then

$$
v(t) \leq y(t), \quad t \in[T, \infty) \cap \mathbb{T}^{\kappa} .
$$

Since $\ominus(-f) \in \mathfrak{R}^{+}$, we have $e_{\ominus(-f)}(\infty, t)>0$. From (3.35) and Lemma 2.2, we obtain

$$
\begin{aligned}
y^{\Delta}(t) & =-[g(t) u(t)+h(t)] \geq-\left[g(t)\left(v(t) e_{\ominus(-f)}(\infty, t)\right)^{\frac{1}{p}}+h(t)\right] \\
& \geq-\left[g(t)\left(\frac{1}{p} K^{\frac{1-p}{p}} v(t) e_{\ominus(-f)}(\infty, t)+\frac{p-1}{p} K^{\frac{1}{p}}\right)+h(t)\right] \\
& \geq-\left[g(t)\left(\frac{1}{p} K^{\frac{1-p}{p}} y(t) e_{\ominus(-f)}(\infty, t)+\frac{p-1}{p} K^{\frac{1}{p}}\right)+h(t)\right] \\
& =H_{1}(t) y(t)-H_{2}(t),
\end{aligned}
$$

where $H_{1}, H_{2}$ are defined in (3.32).

Since $1+\mu(t) H_{1}(t)>0$, we have $H_{1} \in \mathfrak{R}^{+}$, and a suitable application of Lemma 2.1 yields

$$
y(t) \leq y(\infty) e_{\ominus H_{1}}(\infty, t)+\int_{t}^{\infty} H_{2}(s) e_{H_{1}}(t, \sigma(s)) \Delta s
$$


Since $y(\infty)=a(T)$, we obtain

$$
y(t) \leq a(T) e_{\ominus H_{1}}(\infty, t)+\int_{t}^{\infty} H_{2}(s) e_{H_{1}}(t, \sigma(s)) \Delta s .
$$

Combining (3.35), (3.37) and (3.40), we obtain

$$
\begin{aligned}
& u(t) \leq\left\{\left[a(T) e_{\ominus H_{1}}(\infty, t)+\int_{t}^{\infty} H_{2}(s) e_{H_{1}}(t, \sigma(s)) \Delta s\right] e_{\ominus(-f)}(\infty, t)\right\}^{\frac{1}{p}}, \\
& t \in[T, \infty) \cap \mathbb{T}^{\kappa} .
\end{aligned}
$$

Setting $t=T$ in (3.41) yields

$$
u(T) \leq\left\{\left[a(T) e_{\ominus H_{1}}(\infty, T)+\int_{T}^{\infty} H_{2}(s) e_{H_{1}}(T, \sigma(s)) \Delta s\right] e_{\ominus(-f)}(\infty, T)\right\}^{\frac{1}{p}}
$$

Since $T$ is selected from $\mathbb{T}^{\kappa}$ arbitrarily, substituting $T$ with $t$ in (3.42), we obtain the desired inequality (3.31).

Corollary 3.5 Suppose that $\mathbb{T}=\mathbb{R}$. $u, a, f, g, h \in C\left(\mathbb{R}, \mathbb{R}_{+}\right)$, and $a$ is decreasing on $\mathbb{R} . p$ is defined as in Theorem 3.3. If $u(t)$ satisfies the following inequality:

$$
u^{p}(t) \leq a(t)+\int_{t}^{\infty}\left[f(s) u^{p}(s)+g(s) u(s)+h(s)\right] d s, \quad t \in \mathbb{R}
$$

then

$$
\begin{aligned}
u(t) \leq & \left\{\left[a(t) \exp \left(-\int_{t}^{\infty} H_{1}(\tau) d \tau\right)+\int_{t}^{\infty} H_{2}(s) \exp \left(\int_{s}^{t} H_{1}(\tau) d \tau\right) d s\right]\right. \\
& \left.\times \exp \left(\int_{t}^{\infty} f(\tau) d \tau\right)\right\}^{\frac{1}{p}}, \quad t \in \mathbb{R},
\end{aligned}
$$

where $H_{1}(t)=-g(t) \frac{1}{p} K^{\frac{1-p}{p}} \exp \left(\int_{t}^{\infty} f(\tau) d \tau\right)$, and $H_{2}$ is defined as in Theorem 3.3.

Corollary 3.6 Suppose that $\mathbb{T}=\mathbb{Z} . u, a, f, g \in\left(\mathbb{Z}, \mathbb{R}_{+}\right)$, and a is decreasing on $\mathbb{Z}$. $p$ is the same as in Theorem 3.3. If $u(n)$ satisfies the following inequality:

$$
u^{p}(n) \leq a(n)+\sum_{s=n}^{\infty}\left[f(s) u^{p}(s)+g(s) u(s)+h(s)\right], \quad n \in \mathbb{Z},
$$

then

$$
\begin{aligned}
& u(n) \leq\left\{\left\{a(n) \prod_{\tau=n}^{\infty} \frac{1}{1+H_{1}(\tau)}+\sum_{s=n}^{\infty}\left[H_{2}(s)\left(\prod_{\tau=n}^{s} \frac{1}{1+H_{1}(\tau)}\right)\right]\right\} \prod_{\tau=n}^{\infty} \frac{1}{1-f(\tau)}\right\}^{\frac{1}{p}}, \\
& n \in \mathbb{Z},
\end{aligned}
$$

where $H_{1}(\tau)=-g(\tau) \frac{1}{p} K^{\frac{1-p}{p}} \prod_{\xi=\tau}^{\infty} \frac{1}{1-f(\xi)}$, and $H_{2}$ is defined as in Theorem 3.3. 
Theorem 3.4 Suppose that $\sup _{t \in \mathbb{T}^{\kappa}} t=\infty, u, a, f$ are defined as in Theorem 3.1, and $p \geq 1$. $L \in C\left(\mathbb{T}^{\kappa} \times \mathbb{R}_{+}, \mathbb{R}_{+}\right)$, and $0 \leq L(s, x)-L(s, y) \leq M(s, y)(x-y)$ for $x \geq y \geq 0$, where $M \in C\left(\mathbb{T}^{\kappa} \times\right.$ $\left.\mathbb{R}_{+}, \mathbb{R}_{+}\right)$. If $u(t)$ satisfies the following inequality:

$$
u^{p}(t) \leq a(t)+\int_{t}^{\infty}\left[f(s) u^{p}(s)+L(s, u(s))\right] \Delta s, \quad t \in \mathbb{T}^{\kappa},
$$

then

$$
u(t) \leq\left\{\left[a(t) e_{\ominus \widehat{H}_{1}}(\infty, t)+\int_{t}^{\infty} \widehat{H}_{2}(s) e_{\widehat{H}_{1}}(t, \sigma(s)) \Delta s\right] e_{\ominus(-f)}(\infty, t)\right\}^{\frac{1}{p}},
$$

provided that $1-\mu(t) f(t)>0,1+\mu(t) \widehat{H}_{1}(t)>0$, where

$$
\left\{\begin{array}{l}
\widehat{H}_{1}(t)=-M\left(t,\left(e_{\ominus(-f)}(\infty, t)\right)^{\frac{1}{p} \frac{p-1}{p}} K^{\frac{1}{p}}\right)\left(e_{\ominus(-f)}(\infty, t)\right)^{\frac{1}{p}} \frac{1}{p} K^{\frac{1-p}{p}}, \\
\widehat{H}_{2}(t)=L\left(t,\left(e_{\ominus(-f)}(\infty, t)\right)^{\frac{1}{p}} \frac{p-1}{p} K^{\frac{1}{p}}\right), \quad \forall K>0 .
\end{array}\right.
$$

Proof Let

$$
v(t)=a(t)+\int_{t}^{\infty} L(s, u(s)) \Delta s, \quad t \in \mathbb{T}^{\kappa} .
$$

Then

$$
u^{p}(t) \leq v(t)+\int_{t}^{\infty} f(s) u^{p}(s) \Delta s, \quad t \in \mathbb{T}^{\kappa} .
$$

Similar to the process of (3.34) to (3.35), we obtain

$$
u^{p}(t) \leq v(t) e_{\ominus(-f)}(\infty, t), \quad t \in \mathbb{T}^{\kappa} .
$$

Now fix $T \in \mathbb{T}^{\kappa}$. Let $t \in[T, \infty) \cap \mathbb{T}^{\kappa}$, and

$$
y(t)=a(T)+\int_{t}^{\infty} L(s, u(s)) \Delta s, \quad t \in \mathbb{T}^{\kappa} .
$$

Then

$$
v(t) \leq y(t), \quad t \in[T, \infty) \cap \mathbb{T}^{\kappa} .
$$

Since $1-\mu(t) f(t)>0$, we have $\ominus(-f) \in \mathfrak{R}^{+}$, and furthermore $e_{\ominus(-f)}(\infty, t)>0$. From (3.52), (3.54) and Lemma 2.2, we obtain

$$
\begin{aligned}
y^{\Delta}(t)= & -L(t, u(t)) \geq-L\left(t,\left(y(t)\left(e_{\ominus(-f)}(\infty, t)\right)^{\frac{1}{p}}\right)\right) \\
\geq & -L\left(t,\left(e_{\ominus(-f)}(\infty, t)\right)^{\frac{1}{p}} \frac{1}{p} K^{\frac{1-p}{p}} y(t)+\left(e_{\ominus(-f)}(\infty, t)\right)^{\frac{1}{p}} \frac{p-1}{p} K^{\frac{1}{p}}\right) \\
= & -\left[L\left(t,\left(e_{\ominus(-f)}(\infty, t)\right)^{\frac{1}{p}} \frac{1}{p} K^{\frac{1-p}{p}} y(t)+\left(e_{\ominus(-f)}(\infty, t)\right)^{\frac{1}{p}} \frac{p-1}{p} K^{\frac{1}{p}}\right)\right. \\
& \left.-L\left(t,\left(e_{\ominus(-f)}(\infty, t)\right)^{\frac{1}{p}} \frac{p-1}{p} K^{\frac{1}{p}}\right)+L\left(t,\left(e_{\ominus(-f)}(\infty, t)\right)^{\frac{1}{p}} \frac{p-1}{p} K^{\frac{1}{p}}\right)\right]
\end{aligned}
$$




$$
\begin{aligned}
\geq & -\left[M\left(t,\left(e_{\ominus(-f)}(\infty, t)\right)^{\frac{1}{p}} \frac{p-1}{p} K^{\frac{1}{p}}\right)\left(e_{\ominus(-f)}(\infty, t)\right)^{\frac{1}{p}} \frac{1}{p} K^{\frac{1-p}{p}} y(t)\right. \\
& \left.+L\left(t,\left(e_{\ominus(-f)}(\infty, t)\right)^{\frac{1}{p}} \frac{p-1}{p} K^{\frac{1}{p}}\right)\right] \\
= & \widehat{H}_{1}(t) y(t)-\widehat{H}_{2}(t),
\end{aligned}
$$

where $\widehat{H}_{1}, \widehat{H}_{2}$ are defined in (3.49).

We note that the structure of (3.55) is just similar to (3.38). So, following in a similar manner as the process of (3.38)-(3.40), we deduce

$$
y(t) \leq a(T) e_{\ominus \widehat{H}_{1}}(\infty, t)+\int_{t}^{\infty} \widehat{H}_{2}(s) e_{\widehat{H}_{1}}(t, \sigma(s)) \Delta s .
$$

Combining (3.52), (3.54) and (3.56), we obtain

$$
\begin{aligned}
u(t) & \leq\left\{\left[a(T) e_{\ominus \widehat{H}_{1}}(\infty, t)+\int_{t}^{\infty} \widehat{H}_{2}(s) e_{\widehat{H}_{1}}(t, \sigma(s)) \Delta s\right] e_{\ominus(-f)}(\infty, t)\right\}^{\frac{1}{p}}, \\
t & \in[T, \infty) \cap \mathbb{T}^{\kappa}
\end{aligned}
$$

Setting $t=T$ in (3.57), since $T$ is selected from $\mathbb{T}^{\kappa}$ arbitrarily, after substituting $T$ with $t$, we obtain the desired inequality (3.48).

Remark 3.1 If we take $\mathbb{T}=\mathbb{R}$ or $\mathbb{T}=\mathbb{Z}$ in Theorem 3.4, then we obtain another two corollaries, which are similar to Corollaries 3.1-3.6.

\section{Applications}

In this section, we give some applications for the results presented above. In Examples 1 and 3, new explicit bounds for solutions of certain dynamic equations on time scales are derived by the results established, while in Example 2, we deal with the quantitative properties of solutions of a kind of a dynamical equation on time scales.

Example 1 Consider the following dynamic integral equation on time scales:

$$
u^{p}(t)=C+\int_{t}^{\infty} F(s, u(s)) \Delta s, \quad t \in \mathbb{T}^{\kappa},
$$

with the condition $u(\infty)=C$, where $u \in C_{\mathrm{rd}}(\mathbb{T}, \mathbb{R}), p$ is a positive number with $p \geq 1$.

Assume that $|F(t, u)| \leq f(t)|u|+g(t)$, where $f, g, h \in C_{\mathrm{rd}}\left(\mathbb{T}, \mathbb{R}_{+}\right)$, then

$$
|u(t)| \leq\left\{|C| e_{\ominus \widehat{f}}(\infty, t)+\int_{t}^{\infty} \widehat{g}(s) e_{\widehat{f}}(t, \sigma(s)) \Delta s\right\}^{\frac{1}{p}}, \quad t \in \mathbb{T}^{\kappa}
$$

provided $1+\mu(t) \widehat{f}(t)>0$, where $\widehat{f}(t), \widehat{g}(t)$ are defined the same as in Theorem 3.2.

In fact, from (4.1) we have

$$
|u(t)|^{p} \leq|C|+\int_{t}^{\infty}|F(s, u(s))| \Delta s \leq|C|+\int_{t}^{\infty}[f(s)|u(s)|+g(s)] \Delta s .
$$

Then a suitable application of Theorem 3.2 yields the desired result. 
Example 2 Consider the following dynamic integral equation on time scales:

$$
u(t)=C+\int_{t}^{\infty} F(s, u(s)) \Delta s, \quad t \in \mathbb{T}^{\kappa}
$$

where $u$ is the same as in Example 1.

Assume that $\left|F\left(t, u_{1}\right)-F\left(t, u_{2}\right)\right| \leq f(t)\left|u_{1}(t)-u_{2}(t)\right|$, where $f \in C_{\text {rd }}\left(\mathbb{T}, \mathbb{R}_{+}\right)$, then Eq. (4.3) has at most one solution.

In fact, if $u_{1}(t)$ and $u_{2}(t)$ are two solutions to Eq. (4.3), then

$$
\begin{aligned}
\left|u_{1}(t)-u_{2}(t)\right| & =\left|\int_{t}^{\infty} F\left(s, u_{1}(s)\right)-F\left(s, u_{2}(s)\right) \Delta s\right| \leq \int_{t}^{\infty}\left|F\left(s, u_{1}(s)\right)-F\left(s, u_{2}(s)\right)\right| \Delta s \\
& \leq \int_{t}^{\infty} f(s)\left|u_{1}(s)-u_{2}(s)\right| \Delta s .
\end{aligned}
$$

Then a suitable application of Theorem 3.1 yields $\left|u_{1}(t)-u_{2}(t)\right| \leq 0$, which implies $u_{1}(t) \equiv$ $u_{2}(t)$.

Example 3 Consider the following dynamic differential equation on time scales:

$$
\left(u^{p}(t)\right)^{\Delta}=-G(t, u(t)), \quad t \in \mathbb{T}^{\kappa},
$$

with the condition $u(\infty)=C$, where $u \in C_{\text {rd }}(\mathbb{T}, \mathbb{R})$, and $p$ is a constant with $p \geq 1$.

Assume that $|G(t, u)| \leq f(t)|u|^{p}+L(t,|u(t)|)$, where $f, L$ are defined the same as in Theorem 3.4 , then

$$
|u(t)| \leq\left\{\left[|C| e_{\ominus \widehat{H}_{1}}(\infty, t)+\int_{t}^{\infty} \widehat{H}_{2}(s) e_{\widehat{H}_{1}}(t, \sigma(s)) \Delta s\right] e_{\ominus(-f)}(\infty, t)\right\}^{\frac{1}{p}}
$$

provided that $1-\mu(t) f(t)>0,1+\mu(t) \widehat{H}_{1}(t)>0$, where $\widehat{H}_{1}(t), \widehat{H}_{2}(t)$ are the same as in Theorem 3.4 .

In fact, considering $u(\infty)=C$, then the equivalent form of (4.5) is denoted by

$$
u^{p}(t)=C+\int_{t}^{\infty} G(s, u(s)) \Delta s, \quad t \in \mathbb{T}^{\kappa}
$$

Furthermore,

$$
\begin{aligned}
|u(t)|^{p} & \leq|C|+\int_{t}^{\infty}|G(s, u(s))| \Delta s \\
& \leq|C|+\int_{t}^{\infty}\left[f(s)|u(s)|^{p}+L(s,|u(s)|)\right] \Delta s, \quad t \in \mathbb{T}^{\kappa} .
\end{aligned}
$$

Then a suitable application of Theorem 3.4 yields the desired inequality (4.6).

\section{Conclusions}

We have established some new Gronwall-Bellman type integral inequalities on time scales containing integration on infinite intervals, which can be used in the research of boundedness and other qualitative properties as well as quantitative properties of solutions of 
certain dynamic equations on time scales. From the corollaries in Section 3, one can see the established inequalities unify continuous and discrete analysis. Finally, we note that the process of Theorems 3.1-3.4 can be applied to establish delay inequalities with two independent variables on time scales.

Competing interests

The authors declare that they have no competing interests.

\section{Authors' contributions}

ZM and CW carried out the main part of this article. All authors read and approved the final manuscript.

\section{Acknowledgements}

The authors would like to thank the referees very much for their valuable suggestions on improving this paper.

Received: 4 November 2012 Accepted: 27 April 2013 Published: 15 May 2013

\section{References}

1. Hilger, S: Analysis on measure chains - a unified approach to continuous and discrete calculus. Results Math. 18, 18-56 (1990)

2. Bohner, M, Erbe, L, Peterson, A: Oscillation for nonlinear second order dynamic equations on a time scale. J. Math. Anal. Appl. 301(2), 491-507 (2005)

3. Xing, Y, Han, M, Zheng, G: Initial value problem for first-order integro-differential equation of Volterra type on time scales. Nonlinear Anal. TMA 60(3), 429-442 (2005)

4. Agarwal, RP, Bohner, M, O'Regan, D, Peterson, A: Dynamic equations on time scales: a survey. J. Comput. Appl. Math. 141(1-2), 1-26 (2006)

5. Liu, WJ, Li, CC, Hao, YM: Further generalization of some double integral inequalities and applications. Acta Math. Univ. Comen. 77(1), 147-154 (2008)

6. Cheng, XL: Improvement of some Ostrowski-Grüss type inequalities. Comput. Math. Appl. 42, 109-114 (2001)

7. Bohner, M, Matthews, T: The Grüss inequality on time scales. Commun. Math. Anal. 3(1), 1-8 (2007)

8. Ngô, QA: Some mean value theorems for integrals on time scales. Appl. Math. Comput. 213, 322-328 (2009)

9. Liu, WJ, Ngô, QA: Some lyengar-type inequalities on time scales for functions whose second derivatives are bounded. Appl. Math. Comput. 216, 3244-3251 (2010)

10. Saker, SH: Some nonlinear dynamic inequalities on time scales and applications. J. Math. Inequal. 4, 561-579 (2010)

11. Saker, SH: Some nonlinear dynamic inequalities on time scales. Math. Inequal. Appl. 14(3), 633-645 (2011)

12. Li, WN: Some delay integral inequalities on time scales. Comput. Math. Appl. 59, 1929-1936 (2010)

13. $\mathrm{Ma}, \mathrm{QH}$, Pečarić, J: The bounds on the solutions of certain two-dimensional delay dynamic systems on time scales. Comput. Math. Appl. 61, 2158-2163 (2011)

14. Saker, SH: Nonlinear dynamic inequalities of Gronwall-Bellman type on time scales. Electron. J. Qual. Theory Differ. Equ. 2011, 86 (2011)

15. Li, WN: Some new dynamic inequalities on time scales. J. Math. Anal. Appl. 319, 802-814 (2006)

16. Feng, QH, Meng, FW, Zheng, B: Gronwall-Bellman type nonlinear delay integral inequalities on time scales. J. Math. Anal. Appl. 382, 772-784 (2011)

17. Agarwal, RP, Bohner, M, Peterson, A: Inequalities on time scales: a survey. Math. Inequal. Appl. 4(4), $535-557$ (2001)

18. Bohner, M, Peterson, A: Dynamic Equations on Time Scales: An Introduction with Applications. Birkhäuser, Boston (2001)

19. Feng, QH, Meng, FW, Zhang, YM, Zheng, B, Zhou, JC: Some nonlinear delay integral inequalities on time scales arising in the theory of dynamics equations. J. Inequal. Appl. 2011, 29 (2011)

20. Jiang, FC, Meng, FW: Explicit bounds on some new nonlinear integral inequality with delay. J. Comput. Appl. Math. 205, 479-486 (2007)

doi:10.1186/1029-242X-2013-245

Cite this article as: Meng et al.: Some new integral inequalities on time scales containing integration on infinite intervals. Journal of Inequalities and Applications 2013 2013:245. 